\title{
Electrical characterisation of domestic refrigeration compressors
}

\author{
T.A. Kattakayam \\ K.Srinivasan
}

Indexing terms: Refrigerator motors, Energy efficiency

\begin{abstract}
The results of a study on electrical characteristics of hermetic compressor motors used in domestic refrigerators are described. The voltage and current waveforms during start and run states have been investigated for sinusoidal, near square wave and near triangular wave input voltages in the range $170-260 \mathrm{~V}$. An instrumentation system based on an ADC/DAC plug-in card in a personal computer is developed for this purpose. It is deduced that through a considerable redesign of the windings the optimal point of operation corresponding to nominal supply voltage and an improvement in the power factor can be achieved.
\end{abstract}

\section{Introduction}

In the recent past a considerable amount of attention has been paid to domestic refrigerators for, perhaps, two reasons. First, their share in the domestic energy bill is considerable. It is reported that domestic refrigerators and freezers consume $7 \%$ of the energy generated in the US [1] and 2000MW in the UK [2]. Secondly, there is a growing demand to operate them with ozone-friendly working fluids. There have been investigations on improvements of mechancial compressors [3] and cabinet temperature distributions [4]. Test standards are being revised to improve their energy efficiency ratio [5], but it appears that there have been few studies on the motors of these refrigerators.

Parameters affecting the performance of induction motors in general have been highlighted by Singh and Parker [6]. Specific studies on induction start, induction run motors driving a refrigerator compressor are important so that the motor designs can be improved to match the developments in compressor design. The compressor motor always starts on load and is intermittent in nature. The base load arises due to the inertia of the compressor, acceleration of the refrigerant through the system, acceleration of the valve reeds and friction. Further, if the cut-in temperature is reached before the pressure equalisation across the capillary expansion device, then the compressor also has to work against the discharge to suction pressure differential. A current relay is used to switch from the start to the run condition. This paper describes the results of a detailed parametric study on the electrical characteristics of a motor during the starting transient and its approach to stabilised conditions of running.

\section{Experimental set-up}

A schematic diagram of the experimental set-up is shown in Fig. 1. The test specimen is a 1651 top-freezer single-door domestic refrigerator. The supply voltage signal and the induced voltage due to the load current are measured using a fast sampling analogue to digital PC plug-in card. The voltage is measured after stepping down through an isolation transformer (PT) by 20:1. The current drawn by the compressor is measured using two methods. In the first method, a current transformer (CT) with a rating of 25VA is used. The voltage drop across a $0.1 \Omega$ resistor due to the secondary current will represent the current drawn by the motor. In the second method, a high common mode rejection type isolation amplifier (HCPL-7800) is used. A board with this integrated circuit was developed in a sister laboratory of our Institute [7].

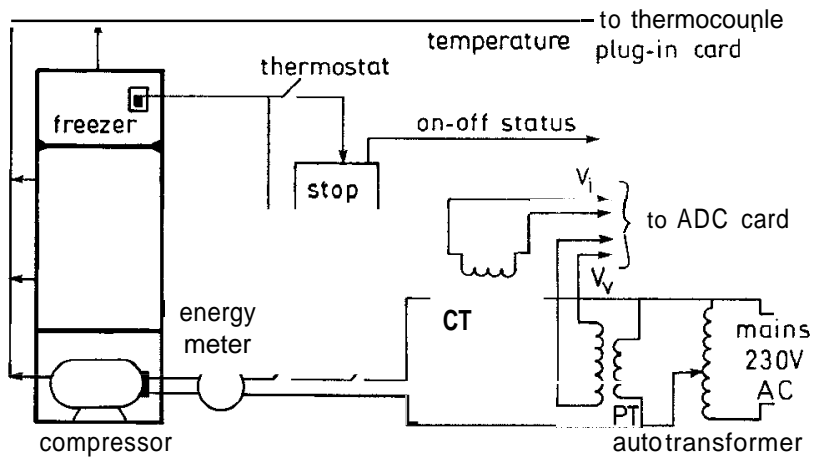

Fig.1 Experimental arrangement

The data acquisition plug-in card consists of a 16channel 12-bit integrating type ADC, two 12-bit DACs, a digital input and an output each of 16 bits with necessary buffers, a programmable gain instrumentation amplifier with a maximum selectable gain of 256 and controls for a PC-AT bus interface. The time base for sampling is derived from a basic $1 \mathrm{MHz}$ crystal clock. A programmable timer controller is sampling rate of $4000 / \mathrm{s}$ in this study.

The transient voltage and current waveforms are tracked for various supply voltages and different time 
delays between stop to start sequences of a refrigerator compressor. The voltage and current channel signals received with the time base are converted to actual conditions using a multiplication factor. This is obtained from a calibration of the RMS value against a Keithley (Model 109) digital multimeter under steady state conditions. The RMS value for the nonsinusoidal waveforms is determined from numerical integration of the acquired waveform. For continuously changing signals the precision of measurements is $2 \%$ for the voltage signals. For current measurement the uncertainties are $\sim 0.1 \mathrm{~A}$, and $0.05 \mathrm{~A}$ by the CT method and the HCPL method, respectively.

For experimental purposes the usual vapour pressure thermometer based thermostat relay in the refrigerator is modified as follows. The open status of this relay activates an external timer such that the latter closes another relay in series with the compressor when the time delay condition is satisfied. A detailed description of this aspect is given elsewhere [8]. Experiments are carried out for time delays between 6 and $15 \mathrm{~min}$ and for input voltages from 160 to $260 \mathrm{~V}$, which is also the specified range of voltages acceptable for safe operation of that compressor. The test conditions are: (i) conventional $50 \mathrm{~Hz}$ mains supply (sinusoidal waveform); (ii) power supply by a $180 \mathrm{Ah}$ battery bank through PWM inverter (near square waveform, $50 \mathrm{~Hz}$ ); and (iii) the output of a petrol/kerosene generator set (near triangular waveform $50 \mathrm{~Hz}$ ). The CT method is used for the first two cases only.

The auxiliary data logged include the steady state voltage and current drawn by the compressor motor, and the on/off status of the refrigerator. The integrated hourly and daily average energy consumption, and cumulative hours of ON time are thereby obtained.

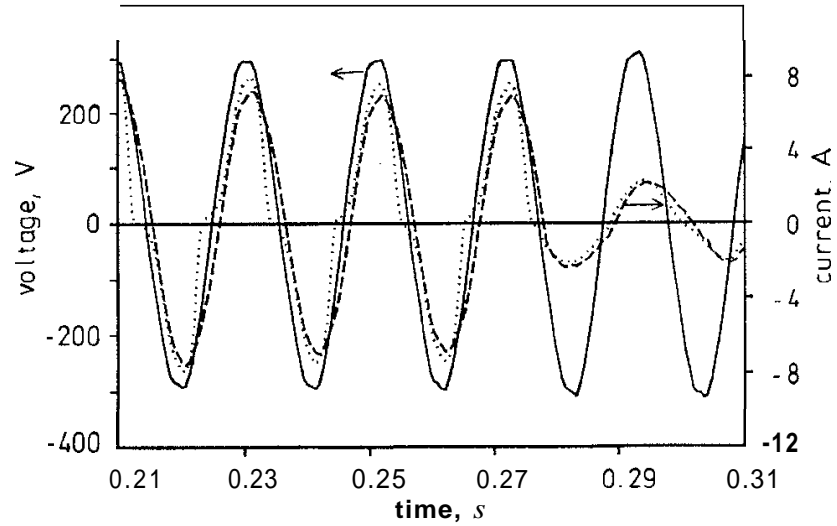

Fig.2 Voltage and current waveforms at transition $220 \mathrm{~V} ; 50 \mathrm{~Hz}$ mains (sinusoidal)

- - - current (HCPL)

.......... current (CT)

\section{Results and discussion}

Figs. 2-4 show typical plots of voltage and current transients at the point of transition from starting to running states for all the three sources of power and an on-time delay of $10 \mathrm{~min}$. Similar plots are also obtained for other input voltages. It may be observed (Fig. 2) that the voltage waveform does not undergo any major distortion during the starting transient. The distortion in the current waveform for the case of the CT method is due to its loading when a large starting current is drawn and the consequent saturation.

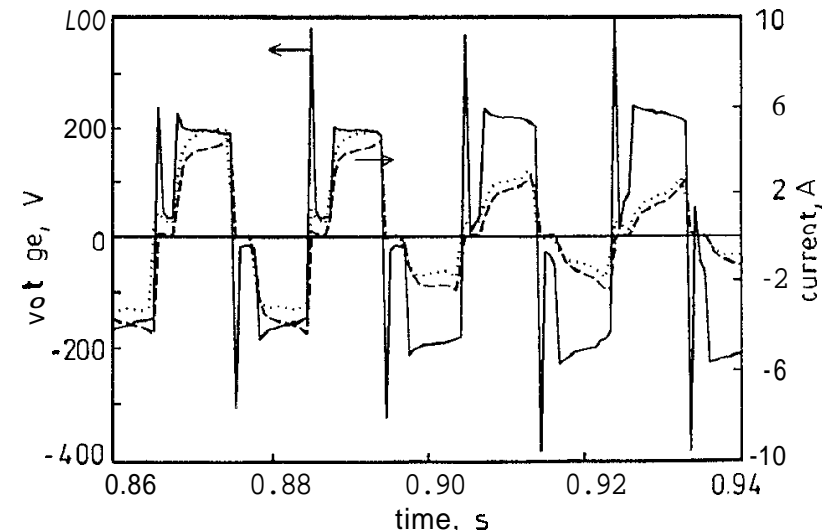

Fig.3 Voltage and current waveforms at transition $220 \mathrm{~V} ; 50 \mathrm{~Hz} P W \mathrm{P}$ inverter (near square wave)

- $\ldots$ - voltage current (HCPL)

........... current (CT)

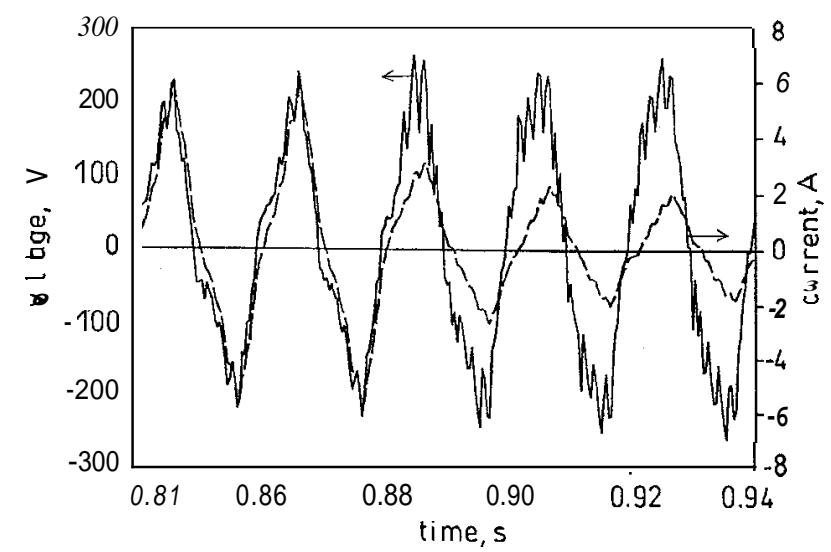

Fig.4 Voltage and current waveforms at transition $220 \mathrm{~V}$; $50 \mathrm{~Hz}$ generator (near triangular wave)

voltage
--- current $(\mathrm{HCPL})$

The rising part of the waveform is measured with reasonable accuracy, but after saturation at the peak current there is a steep descent in the signal as measured by it. The steepness of fall decreases as the magnitude of the current decreases. The peak currents measured by both the methods are in good agreement. The sinusoidal wave of the current signal under running conditions is satisfactorily measured by the CT method. A complete current transient for sinusoidal input obtained from the HCPL is shown in Fig. 5.

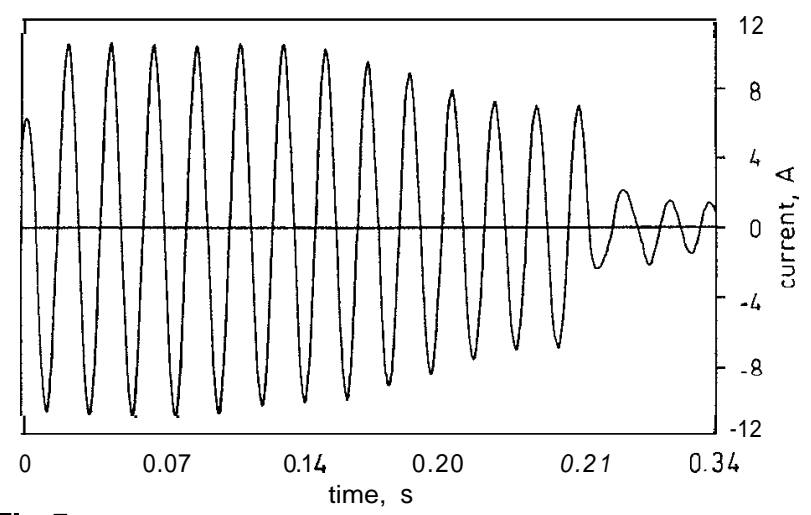

Fig.5 Complete transient current waveform for $220 \mathrm{~V}$ mains, $10 \mathrm{~min}$ time delay

In the case of a generator as a source (Fig. 4) the waveform is close to being triangular. The harmonics on the voltage wave indicate asynchronous rotation of the engine shaft. In all the three cases it was observed 
that the running current decreases further and reaches a stable value only after about $6-10$ s of change over from start to run states.

The discrepancy associated with the CT method can be reduced by opting for a higher VA rating, but this would diminish the sensitivity under steady state operating conditions. The CT method is somewhat less accurate than the HCPL method because of the hysteresis effects of the winding and phase shift errors. However, peak currents drawn during start and steady state energy evaluation - a cost effective, though less precise, CT method-may be adequate. A higher rating of the CT could alleviate the problem, but then the steady state resolution will be poorer. For a precise analysis of the motor windings the HCPL method may be more apt.

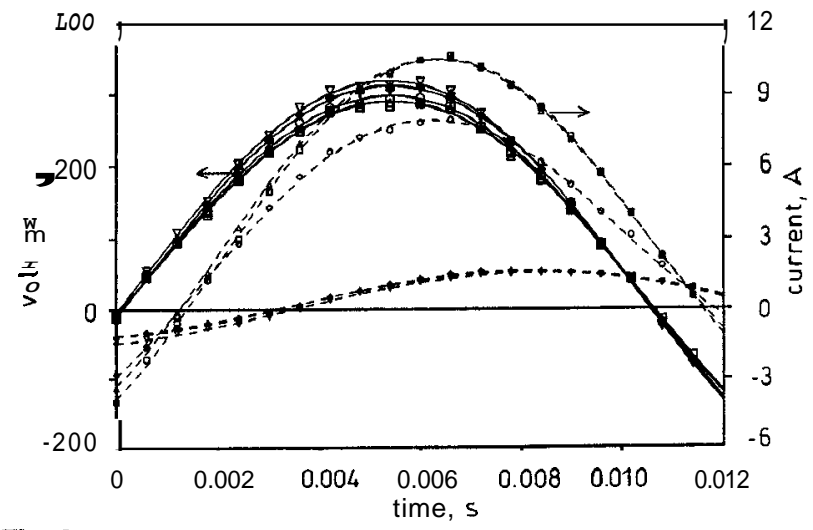

Fig.6 Current and voltage waveforms during and after start (mains) voltae

$\square 37 \mathrm{~ms} ;$ A $120 \mathrm{~ms} ;$ O $204 \mathrm{~ms} ; \nabla 371 \mathrm{~ms} ;+1.160 \mathrm{~s} ;$ O $1.642 \mathrm{~s}$

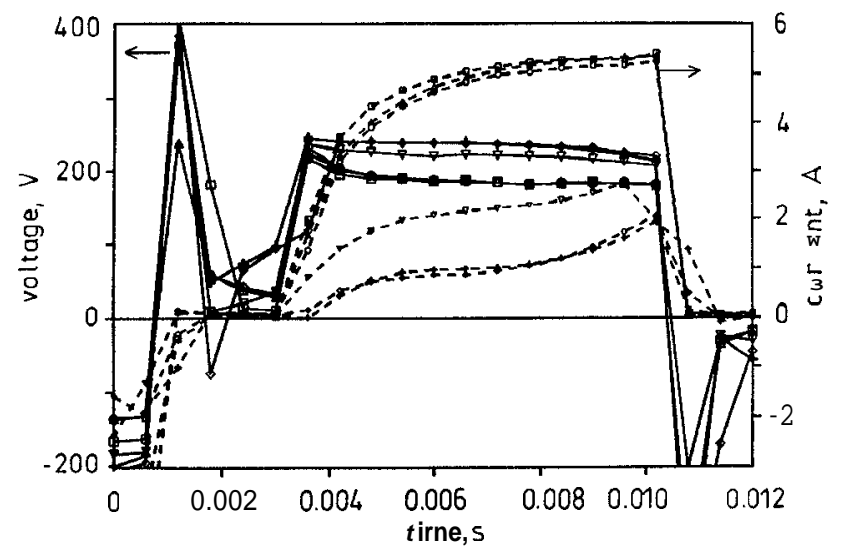

Fig. 7 Current and voltage waveforms during and after start (inverter) $\underline{\text { Fig. }}$ voltage

$\square$ 37ms; A $120 \mathrm{~ms} ;$ O 204ms; $\nabla 371 \mathrm{~ms} ;+1.160 \mathrm{~s} ;$ O $1.642 \mathrm{~s}$

Figs. 6 and 7 show the voltage and current waveforms during the transient (from HCPL data) for $220 \mathrm{~V}$ nominal input for mains and inverter operation, respectively. The time base (abscissa) is normalised to show one positive half-cycle. Fig. 6 shows virtually no distortion of the current waveform, indicating uniformity in the inductance of the winding. The interval between negative to positive zero crossings is averaged to evaluate the frequency of the power supply and hence the cycle time for the voltage waveform. The time difference between zeros of voltage and current waveforms is used to determine the power factor in the case of sinusoidal input. The power factor is high during the starting transient but decreases to 0.67 after the running condition has stabilised. This value is low but can be improved marginally by reducing the supply voltage after starting. Fig. 7 shows a marked change in the current waveform of the PWM inverter after the running condition is reached. The convexity during the start transforms to concavity later. This is an indication of the saturation of the core. The magnitude of the voltage increases after the transition because of reduced load current.

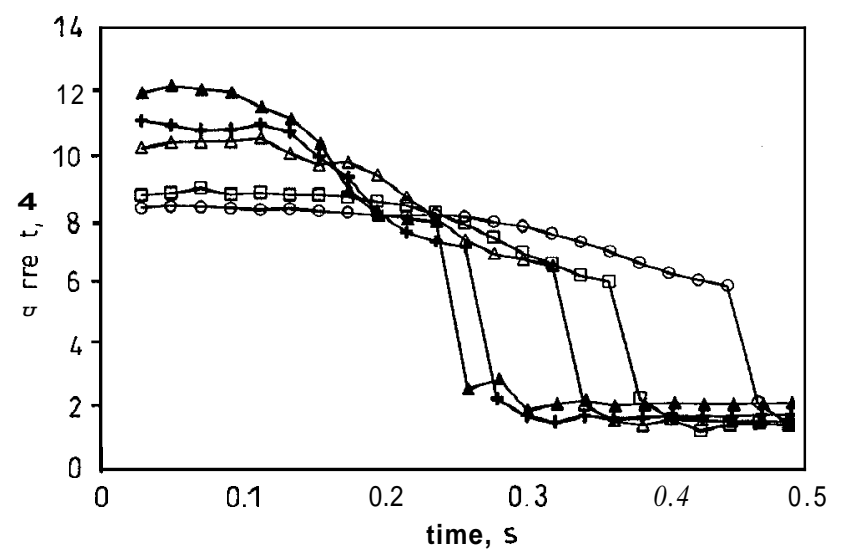

Fig.8 Transient trace of peak currents drawn by motorfor mains O $170 \mathrm{~V}$ $\triangle 210 \mathrm{~V}$ $+230 \mathrm{~V}$ A $250 \mathrm{~V}$

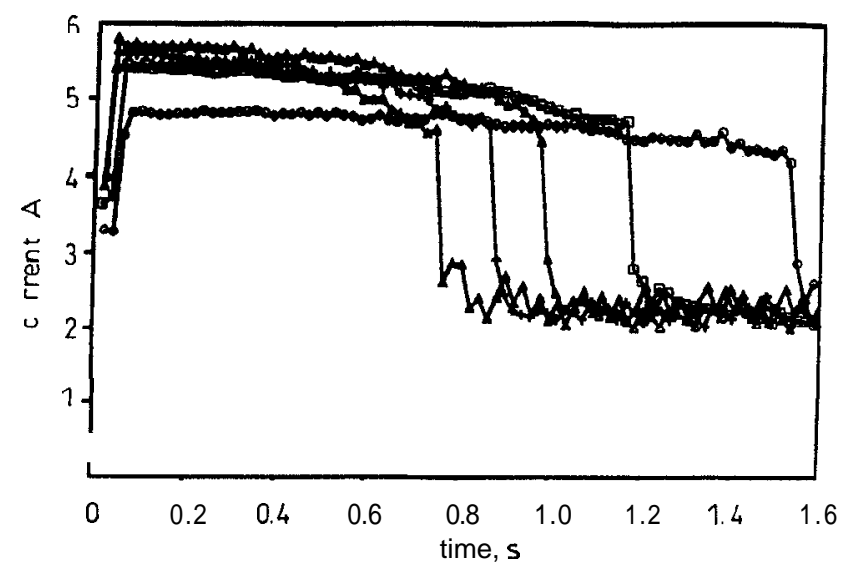

Fig.9 Transient trace of peak currents drawn by motor for PWM inverter $\mathrm{O} 170 \mathrm{~V}$

$\square 190 \mathrm{~V}$

$\mathrm{A} 210 \mathrm{~V}$
$+230 \mathrm{~V}$

A $250 \mathrm{~V}$

The locus of the peaks of the current waveform is shown in Fig. 8, which indicates that the peak current drawn by the motor reaches a high value of $12 \mathrm{~A}$ under certain input conditions. Fig. 9 is a similar plot for an inverter waveform, but here the peak current corresponds to the part of the waveform excluding the spikes. Both these Figures are for an on-time delay of $10 \mathrm{~min}$. It is evident that the starting transient decreases with an increase in the supply voltage. A square wave inverter used here has a 750VA transformer and a $1.2 \mathrm{kVA}$ transistor drive and is powered by a $24 \mathrm{~V}$ battery bank charged by a field of solar photovoltaic panels. The peak currents could be limited to $6 \mathrm{~A}$ but at the expense of an increase in the starting transient. The ratings of the power transistors and consequent parasitic power losses were also reduced without sacrificing the motor performance. Further, the 
inverter has been configured to provide a high supply voltage of $\sim 260 \mathrm{~V}$ to take advantage of a short starting transient. The voltage is reduced to a level of $230 \mathrm{~V}$ after the running condition sets in. This is the voltage at which the power consumed by the motor is the minimum (Fig. 10). The average power in this Figure was calculated based on daily energy consumption (kWh)/ 24. It can be seen that if the voltage supply can be stabilised at the optimal value considerable energy saving can be realised even in existing refrigerators. The CT method described here can be used to locate the optimal operating point.

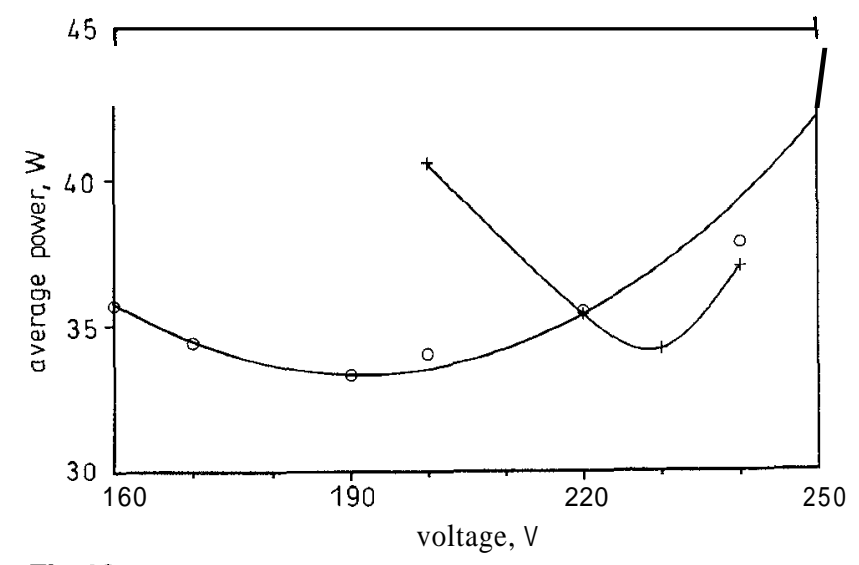

Fig.10 Power consumptionpatterns $O$ mains

With the inverter operation the power supply had to be designed to provide an output voltage of $250-260 \mathrm{~V}$, $230 \mathrm{~V}$ level after running conditions have stabilised and OV during the off state of the refrigerator irrespective of the status of the battery bank voltage. In our case the battery bank is charged from a field of photovoltaic collectors. A control circuit is operated through the DAC channel of the plug-in card to manipulate the base current for the driver transistors of the inverter to realise an output voltage in accordance with the above needs. This has resulted in an additional energy saving on the refrigerator in the uninterrupted operation mode [8]. The time delay also enables the transistor bank to cool down during the off state of the refrigerator. The temperature mapping of the interior of the refrigerator (outside the perview of this paper) has been carried out which shows that the time delay has no effect on the internal distribution [9].

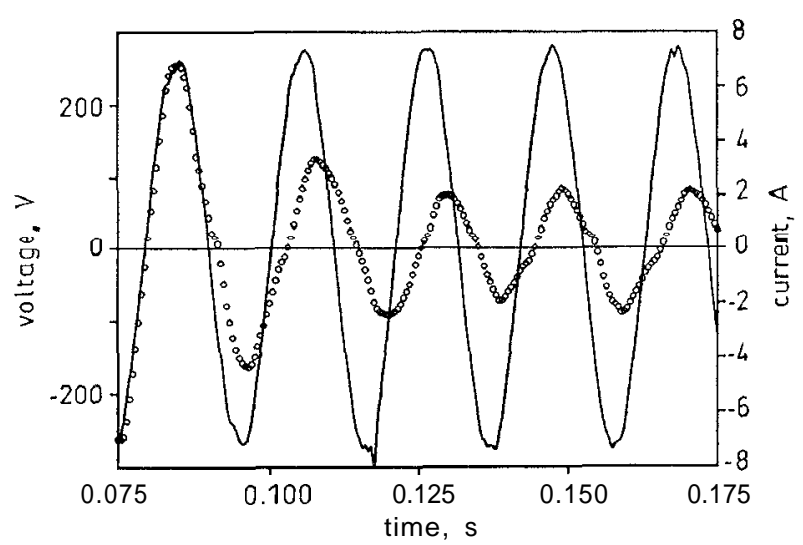

Fig.11 Voltage and current waveformsfor another compressor motor $\bar{O}$ voltage
In view of the predictions [10] that compressors used with new refrigerants need more energy, the methods described here could assist in obtaining data for redesigning the motor windings for minimal electrical losses and optimal mechanical performance. The HPCL method can be adopted by manufacturers for tests on prototypes. For example, the windings of the motor studied here appear to be optimised for a mains supply voltage of $\sim 180-200 \mathrm{~V}$, whereas it should have been for $220 \mathrm{~V}$. The motor windings can be improved to realise a power factor higher than 0.67 . For a second hermetic compressor motor of a different capacity and from another country, Fig. 11 shows that: (i) the starting transient is as short as $90 \mathrm{~ms}$; (ii) there is a distortion in the current waveform when operating on the mains; and (iii) the power factor is nearly unity during the start but decreases considerably soon after. This again shows that the windings have to be improved considerably.

\section{Conclusions}

An experimental parametric study on the start and run states of an induction motor in a hermetic refrigeration compressor has been conducted. Based on the results it is observed that energy conservation even in existing refrigrators is possible provided the operating voltage can be controlled. An on-time delay is essential to reduce the magnitude of peak currents drawn by the compressor. The compressors can be safely powered by near square wave inverters or even triangular wave sources. The studies indicate that the starting and the running windings need redesigning to augment energy efficiency and to improve the power factor of the motor from the existing level of -0.7 .

\section{Acknowledgments}

The authors thank Professor V. Ramanarayanan for providing the PCB containing the HPCL current transducer. The work reported herein was supported through a grant from the Department of Science and Technology, Government of India.

\section{References}

1 MEIER, A.: 'Refrigerator energy use in the laboratory and in the field', Energy Build., 1995, 22, pp. 233-243

2 HERRING, H.: 'Is Britain a Third World country?, The case of German refrigerators', Energy Policy, 1994, 22, pp. 779-787

3 VOLLMER, D., GUNTHER, E., and MEYER, A.: 'Progress in the science and technology of refrigeration in food engineering' (Int. Inst. Refrig., Paris, 1990), p. 133

4 JAMES, S.J., and EVANS, J.A.: 'Performance of domestic refrigeration', Proc. Inst. Refrig., 1990-91, 87, pp. 31-40

5 BANSAL, P.K., and KRUGER, R.: 'Test standards for household refrigerators and freezers: preliminary comparisons', Znt. $J$. Refrig., 1995,18, pp. 4-20

6 SINGH, C., and SARKAR, D.: 'Practical considerations in the optimization of induction motor design', IEE Proc. $B, 1992,139$, pp. $365-373$

7 RAMPRASAD, D., and RAMANARAYAN, V.: Project report, Department of Electrical Engineering, Indian Institute of Science, Bangalore

8 KATTAKAYAM, T.A., and SRINIVASAN, K.: 'Photovoltaic panel-generator based autonomous power source for small refrigeration units', Sol. Energy, 1996, 56, pp. 543-552

9 KATTAKAYAM, T.A.: 'Studies on autonomous photovoltaic small refrigeration systems'. PhD thesis, Department of Mechanical Engineering, Indian Institute of Science, Bangalore, 1996

10 SRINIVASAN, K.: 'A comparative study of vapour compression refrigeration working fluids, in the wake of the Montreal Protocol', J. Inst. Energy, 1994, 67, pp. 109-112 